\title{
The Third Meeting on Bone Quality, June 2008
}

\author{
C. L. Benhamou
}

Published online: 2 April 2009

(C) International Osteoporosis Foundation and National Osteoporosis Foundation 2009

Bone strength is dependent on bone mass and bone quality. Among the so-called qualitative factors, the size and shape, the cortical properties, and the microstructural arrangement of trabecular bone play a role which has been studied at the previous annual French Bone Quality Seminars. One quality controlling bone strength is intuitively very important: the quality linked to the material properties.

Everybody knows that the same object, with the same shape and size, falling from the same height will be broken or not depending on its material composition. In other terms, the material properties directly determine the stiffness, brittleness, toughness, elasticity, and ductility.
All these properties are, in bone tissue, conditioned by internal properties of the collagen matrix and of the bone crystal and are dependent on the bone remodeling process. Some properties, such as the vascular richness or the quantity of fat tissue in cancellous bone, may play a role which is poorly defined at this time, and not easy to characterize. However, more and more explorations are being developed in order to evaluate the ultrastructural parameters and material properties of bone tissue.

It is the purpose of these papers from the Third Meeting on Bone Quality to detail these explorations.

Data presented at the Third Meeting on Bone Quality, France, 24-25 June 2008: Bone Ultrastructure.

C. L. Benhamou $(\square)$

Inserm Unit U658, CHR d'Orléans,

Orléans Cedex, France

e-mail: claude-laurent.benhamou@chr-orleans.fr 